\title{
Time evolution of damage due to environmentally assisted aging in a fiber bundle model
}

\author{
S. Lennartz-Sassinek ${ }^{*}$ and I. G. Main \\ School of Engineering and School of Geosciences, University of Edinburgh, Edinburgh, United Kingdom \\ Z. Danku and F. Kun \\ Department of Theoretical Physics, University of Debrecen, Debrecen, Hungary
}

(Received 7 February 2013; published 4 September 2013)

\begin{abstract}
Damage growth in composite materials is a complex process which is of interest in many fields of science and engineering. We consider this problem in a fiber bundle model where fibers undergo an aging process due to the accumulation of damage driven by the locally acting stress in a chemically active environment. By subjecting the bundle to a constant external load, fibers fail either when the load on them exceeds their individual intrinsic strength or when the accumulated internal damage exceeds a random threshold. We analyze the time evolution of the breaking process under low external loads where aging of fibers dominates. In the mean field limit, we show analytically that the aging system continuously accelerates in a way which can be characterized by an inverse power law of the event rate with a singularity that defines a failure time. The exponent is not universal; it depends on the details of the aging process. For localized load sharing, a more complex damage process emerges which is dominated by distinct spatial regions of the system with different degrees of stress concentration. Analytical calculations revealed that the final acceleration to global failure is preceded by a stationary accumulation of damage. When the disorder is strong, the accelerating phase has the same functional behavior as in the mean field limit. The analytical results are verified by computer simulations.
\end{abstract}

DOI: 10.1103/PhysRevE.88.032802

PACS number(s): 46.50.+a, 95.75.Wx, 89.75.Da, 64.60.av

\section{INTRODUCTION}

The brittle failure of materials is an important issue in physics, engineering, and geosciences [1]. In linear elastic fracture mechanics theory, a single fracture nucleates at a critical load in a homogeneous continuum and then propagates in an unstable manner. However, even seemingly homogeneous materials contain many dislocations, flaws, and other heterogeneities. This inherent material disorder makes the approach to dynamic failure much more complex in most materials [2]. Typically, dynamic failure in disordered materials is preceded under steadily increasing load by microcracks nucleating initially randomly throughout the sample, before growing independently until their local stress fields interact and they begin to coalesce. As a consequence, in highly disordered materials, macroscopic brittle failure occurs as the culmination of the gradual accumulation of internal damage. The nucleation and dynamic propagation of cracks generate acoustic emissions (AE), which can be recorded in the form of crackling noise. The measurement of crackling noise is currently one of the main sources of information about the microscopic dynamics of the fracture of heterogeneous materials [3,4].

Geomaterials often suffer constant loads below their fracture strength which may lead to failure in a finite time. Such creep rupture processes play a crucial role in the appearance of natural catastrophes such as landslides and snow avalanches. Acoustic monitoring is one method used to forecast the imminent failure event. However, making a reliable and accurate forecast still remains an open fundamental problem. Due to the decisive role of disorder, theoretical approaches

\footnotetext{
*lennartzsassinek@gmail.com; Present address: Institute for Geophysics and Meteorology, University of Cologne, Cologne, Germany.
}

to the problem are typically based on discrete models such as fiber bundles ([5-10], and references therein) or lattices of springs [11], beams [12,13], and fuses [14]. In Monte Carlo and molecular dynamics simulations of such discrete models, the breaking of a single cohesive element can trigger an entire avalanche of failure events, which corresponds to the acoustic emissions observed in experiments [3,15-17]. However, it has been shown by Lockner that fewer than $0.2 \%$ of the actual damage in a loaded specimen is accompanied by a detectable acoustic emission [3]. The major part of damage is silent, i.e., it is not accessible by AE techniques. Such silent damage has a remarkable effect on the stability and time evolution of the loaded system. Despite its potential importance in understanding the evolution of time-dependent deformation in engineering and geomaterials, such silent damage is difficult to capture in discrete stochastic models of fracture.

In this paper, we investigate the time evolution of silent and acoustic damage accumulating in heterogeneous materials under a constant external load, similar to the work presented in [18]. We consider a recently introduced fiber bundle model [13,19-22], where loaded fibers undergo an aging process accumulating damage and which is a generalization of the model used in [18]. The damage mechanism captures environmentally assisted aging processes, including chemical degradation and stress corrosion cracking. In the model, aging fibers break in a "silent" way, gradually increasing the load on the remaining intact fibers. After some damage events, the local load on fibers may exceed their failure strength, leading to immediate breaking, which in turn can trigger an entire avalanche of breakings in a short time. These dynamic cascades represent potential sources of acoustic emissions. The main advantage of the model is that it provides an explicit representation of both the silent and dynamic (acoustic) components of the total damage in such a way that the dynamic mode is nucleated by the silent one. Similar to [18], we 
carried out analytical calculations and computer simulations of the fiber bundle model, focusing on the silent component of damage, which dominates the evolution of the creeping system at low external loads. The results here are not only a generalization of those presented in [18], but we also corrected a wrong assumption made in the analytical approximations. In addition, in order to understand the role of the spatial range of stress redistribution in the aging process, here we compare the results of the model with strongly localized load redistribution around failed fibers to the mean field limit case of the model.

\section{FIBER BUNDLE MODEL OF AGING DRIVEN FAILURE}

One of the most fundamental models for the failure of heterogeneous materials is the fiber bundle model (FBM) [23,24], which envisages a material as a set of parallel fibers with identical elastic properties but with stochastic failure thresholds $[5,6$, 23-25]. Under external loading, when the local stress exceeds its failure threshold $\sigma_{t h}$, the fiber breaks irreversibly and its load is redistributed over the remaining intact fibers. In the case of equal load sharing (ELS), all fibers keep the same load so that no spatial correlations can arise in the bundle. ELS provides the mean field limit of FBMs, allowing for the analytical investigation of the problem as well. In the more realistic case of localized load sharing (LLS), the load of a broken fiber is equally redistributed solely in the local neighborhood, which gives rise to high stress concentration in the vicinity of failed regions. LLS FBMs describe material failure in situations where the range of internal stresses is limited, e.g., by the vicinity of a free surface [26,27], and their behavior is significantly more complicated to analyze. During the past two decades, several methods have been proposed to incorporate time dependence in FBMs under creep loading conditions: viscoelasticity of fibers [28], complex rheological behavior [4,29], and damage enhanced creep [30-33] have been considered.

Recently, we introduced a further extension and generalization of FBMs incorporating an additional aging mechanism of fibers, which can be relevant for geomaterials [13,18-22]. The aging process represents a large collection of environmentally assisted degradation phenomena, such as chemical degradation, thermally induced cracking, and stress corrosion, which may play an important role in subcritical failure. In this model, each fiber has an internal weakness $c$ so that it can break for two reasons: (i) the local stress $\sigma$ reaches the random strength $\sigma_{t h}$ or (ii) the internal weakness $c$ reaches the disordered critical age $c_{t h}$ of the fiber. We assume that a single fiber ages according to the law

$$
c(t)=a \int_{0}^{t} \sigma^{\gamma}\left(t^{\prime}\right) d t^{\prime},
$$

with $\sigma\left(t^{\prime}\right)$ being the time-dependent local stress of the fiber. In Eq. (1), the rate of aging is mainly controlled by the exponent $\gamma$, while $a$ is a scale parameter. When a fiber breaks due to its age, the local stress again is redistributed according to the ELS or LLS schemes. Under LLS conditions, fibers may get isolated as rupture proceeds, e.g., when broken clusters merge. To redistribute the load at the breaking of such fibers, the size of the affected area was gradually increased, keeping the shape fixed, until it contained at least one intact fiber which received the load. More details on the implementation of the
LLS model can be found in Ref. [22]. The heterogeneity of materials is represented by the randomness of the threshold values $\sigma_{t h}$ and $c_{t h}$. For the mechanical strength $\sigma_{t h}$, we assume a uniform distribution between 0 and 1 in dimensionless units, while for the aging thresholds $c_{t h}$, a uniform distribution is used between the limits $1-W$ and $1+W$. In this way, we can control the amount of damage disorder by varying $W$ in the range $0 \leqslant W \leqslant 1$.

We consider a bundle of $N$ fibers organized on a square lattice of side length $L$. Under a subcritical external load $\sigma<$ $\sigma_{c}$, where $\sigma_{c}$ is the fracture strength of the bundle, an amount of approximately $\sigma N$ fibers initially breaks because their stress $\sigma$ exceeds the local failure threshold $\sigma_{t h}$. The fracture strength $\sigma_{c}$ of LLS fiber bundles has a logarithmic size effect, which was considered carefully in Ref. [22]. For a system of size $L=401$, the critical load is $\sigma_{c}=0.149 \pm 0.0005$, which was determined by independent simulations. The fracture strength of the corresponding ELS bundle is $\sigma_{c}=0.25$. The time evolution of the bundle starts from this configuration, which contains broken fibers randomly scattered over the lattice. Since aging proceeds slowly, it breaks the fibers one by one, gradually increasing the load on the surviving intact fibers. After a certain number of aging-induced breakings, the accumulated load increment becomes sufficient to induce the immediate breaking of a fiber, which in turn can even trigger an avalanche of breakings. The sudden outbreaks of avalanches are analogous to the acoustic events in real measurements, while the aging-induced breaking represents the silent component of the total damage [34]. It follows that the silent damage has a very important effect on the time evolution of the creeping system. The amount and size of triggered avalanches increase significantly with increasing applied stress. In the present paper, we focus on the silent component of damage, i.e., we restrict our analysis to the low load limit of creep failure and to times far away from the failure time where triggered acoustic events can be neglected as an approximation.

\section{CREEP FAILURE IN ELS FBM}

In the following, all quantities related to the ELS case will have a superscript $E$ to distinguish them from the LLS quantities discussed later which all will have the superscript $L$.

After initially subjecting a finite bundle of $N$ fibers to a constant external load $\sigma$, some $\sigma N$ fibers break immediately. If the load is sufficiently small, no subsequent avalanche is triggered. The time evolution of the system starts from this partially failed configuration. Under equal load sharing conditions, each fiber carries the same stress, so no spatial correlation can emerge in the system. After breaking $\sigma N$ fibers, the initial effective stress per fiber is

$$
\sigma_{0}=\sigma \frac{N}{N-\sigma N} \approx \sigma,
$$

where the approximation holds for small applied stresses $\sigma$ compared to the fracture strength $\sigma_{c}$ of the bundle $\sigma \ll \sigma_{c}$. Thus the first fiber breaks due to aging at time $t_{1}$ when the age $c$ of all fibers reaches the minimum value $1-W$, i.e.,

$$
1-W \approx a \sigma^{\gamma} t_{1}
$$


which is equivalent to

$$
t_{1} \approx \frac{1-W}{a \sigma^{\gamma}} .
$$

As long as triggered events can be neglected, the average spacings $\Delta c_{t h}$ of the critical ages $c_{t h}^{i}(i=1, \ldots, N-\sigma N)$ of the remaining intact fibers is

$$
\Delta c_{t h}=\frac{2 W}{N-\sigma N} \approx \frac{2 W}{N} .
$$

After the breaking of the $i$ th fiber, the stress of intact fibers is renormalized to

$$
\sigma_{i}=\sigma \frac{N}{N-\sigma N-i} \approx \sigma \frac{N}{N-i} .
$$

Thus the interevent time $\tau_{i}$ between the breaking of the $i$ th and $(i+1)$ th fibers can simply be obtained as

$$
\tau_{i}=t_{i+1}-t_{i} \approx \frac{2 W}{N a \sigma_{i}^{\gamma}} \approx \frac{2 W(N-i)^{\gamma}}{a \sigma^{\gamma} N^{\gamma+1}},
$$

which is a decreasing function of the event number $i$. This equation is only valid when triggered events can be neglected. Otherwise the stress would be renormalized according to all broken fibers including the triggered ones which results in effectively smaller interevent times. Starting from the expression of $\tau_{i}$, the breaking time $t_{i}$ of the $i$ th fiber can be cast in the form

$$
\begin{aligned}
t_{i} & =t_{1}+\sum_{j=1}^{i-1} \tau_{j} \\
& \approx t_{1}+\frac{2 W}{a \sigma^{\gamma} N^{\gamma+1}} \int_{1}^{i}(N-j)^{\gamma} d j \\
& \approx t_{1}+\frac{2 W}{a \sigma^{\gamma}} \frac{1}{\gamma+1}\left[1-(1-i / N)^{\gamma+1}\right] .
\end{aligned}
$$

It follows for the total amount of damage $D^{E}$ of the bundle under equal load sharing that

$$
D^{E} / N=i / N=1-\left[1-\left(t-t_{1}\right) \frac{(\gamma+1) a \sigma^{\gamma}}{2 W}\right]^{\frac{1}{\gamma+1}},
$$

with $t=t_{i}$ the elapsed time and $t_{1}$ given by Eq. (4). As a consequence, the damage rate $n^{E}(t)$ is

$$
\begin{aligned}
n^{E}(t) & =\frac{d D^{E}}{d t} \\
& =\frac{a \sigma^{\gamma} N}{2 W}\left[1-\left(t-t_{1}\right) \frac{(\gamma+1) a \sigma^{\gamma}}{2 W}\right]^{-\frac{\gamma}{\gamma+1}} .
\end{aligned}
$$

This reduces to the form

$$
n(t) \sim\left(t_{f}-t\right)^{-\frac{\gamma}{\gamma+1}},
$$

where $t_{f}$ denotes the time of macroscopic failure of the system,

$$
t_{f}=[1-W+2 W /(\gamma+1)] /\left[a \sigma^{\gamma}\right] .
$$

This result demonstrates that if the load is equally redistributed over the intact elements, the damage process continuously accelerates as an inverse power law of the event rate to a singularity at the failure time. The exponent, however, is not universal: it depends on the details of the aging process captured in the term $\gamma$. A similar expression to (11), sometimes referred to as the "time-reversed Omori law," has been suggested for the increase in event rate seen before some natural earthquakes and in stacked seismic sequences prior to the mainshock time [35-38]. It is not yet clear if this apparent precursor is a true nucleation phenomenon, as examined here, or an emergent property of the conventional epidemic-type aftershock sequence (ETAS) model [36]. In general, the exponent $p^{\prime}<1$ for the reverse Omori law (implying $\gamma>0$ ), whereas the Omori exponent for aftershock sequences is $p \sim 1$.

An interesting advantage of our discrete approach is that besides the evolution of the cumulative damage, we can also determine the probability distribution $p(\tau)$ of waiting times $\tau$ between consecutive events. From Eq. (7), due to the acceleration of the aging process, the consecutive waiting times $\tau_{i}$ are monotonically decreasing with increasing event number $i$. This has the consequence that the cumulative distribution $P$ of waiting times can be obtained as $P(\tau)=$ $1-i / N$, from which the probability density follows as

$$
p(\tau) \sim \tau^{-(1-1 / \gamma)}
$$

So, the waiting times are also power-law distributed with a $\gamma$ dependent exponent. In the limit of large $\gamma$, both the exponent of the damage rate and of the waiting time distribution converge to one. The same functional form given by Eq. (13) has been presented in Ref. [19] for the probability density of waiting times when damage is the only source of breaking. The closed analytic results obtained for the interevent times, event rates, and accumulated damage demonstrate the advantages of our discrete approach.

In order to numerically verify the above analytical results, we carried out computer simulations of the process of creep rupture. In the simulations, a single bundle of $N=10^{7}$ fibers is considered, subject to a low load $\sigma / \sigma_{c}=10^{-6}$ (for uniformly distributed breaking thresholds $\sigma_{t h}$ between 0 and 1).

The resulting damage rate has a power-law dependence on the time to failure (Fig. 1), where the value of the exponent is in very good agreement with the analytical prediction. No deviation from the power law can be observed. Since each aging-induced breaking increases the stress on the surviving fibers, the system homogeneously accelerates right from the beginning. Figure 2 shows the lifetimes of the systems as a

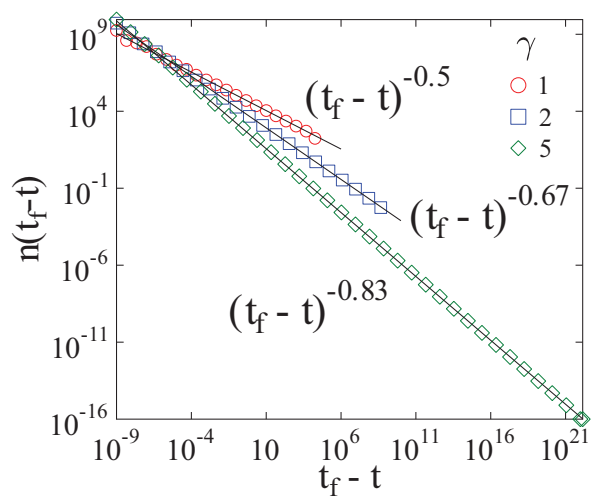

FIG. 1. (Color online) Damage event rate $n$ as a function of time to failure $t_{f}-t$ for different exponents $\gamma$ of the damage law. The results were obtained by ELS simulations of single samples with $10^{7}$ fibers. The straight lines show the analytical results of Eq. (11) for comparison. 


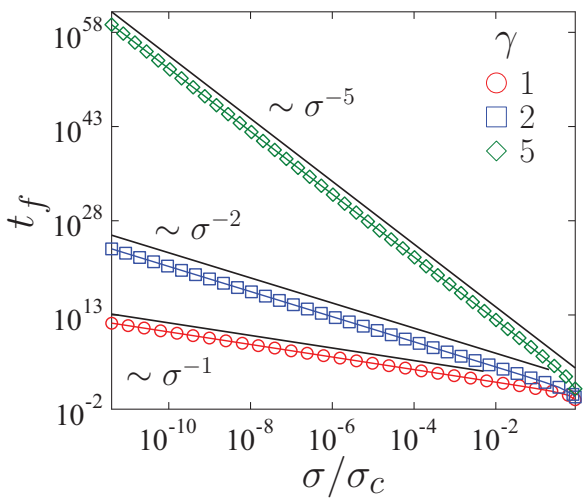

FIG. 2. (Color online) Lifetime $t_{f}$ of samples as a function of load for different values of $\gamma$. The continuous lines represent power laws of exponent $\gamma$. Excellent agreement can be observed with the analytic predictions [Eq. (12)]; slight deviations occur solely in the vicinity of the critical load. The offset between the theoretical lines and the synthetic data is cosmetic (so the lines can be seen).

function of the applied stress. The simulated values show the power-law dependence as predicted in Eq. (12). Only near the critical stress do deviations occur due to the large amount of triggered damage. Here the actual lifetimes are shorter. Thus, for small applied stresses where the creep behavior is dominating, one can use Eq. (12) for $t_{f}$ to fit the power laws for the damage rate shown in Fig. 1. Under circumstances where the real failure time is not known, it is hardly possible to fit the correct power law; see, e.g., [39].

Waiting time distributions are presented in Fig. 3 for the same system as in Fig. 1. Power-law distributions are obtained followed by an exponential cutoff. The value of the exponent is in good agreement with the analytical prediction of Eq. (13), also shown in the figure for the same values of $\gamma$. The small positive deviations of $p(\tau)$ from the power-law behavior for very small $\tau$ can be associated with the presence of triggered events near failure, which have been neglected in the above derivation of Eq. (13).

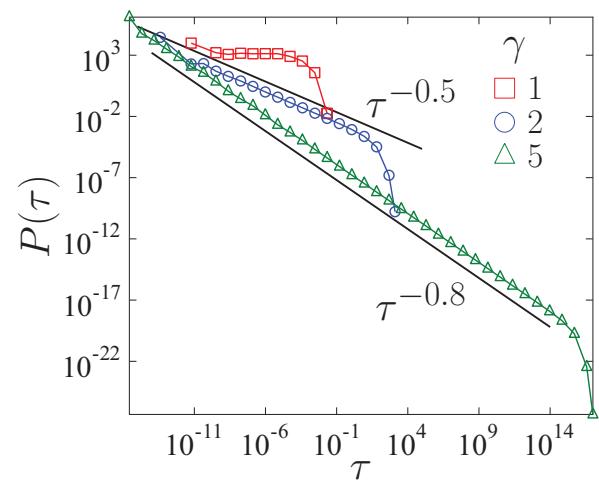

FIG. 3. (Color online) Waiting time distributions obtained by three ELS simulations of a single sample with $10^{7}$ fibers corresponding to different values of $\gamma$ as indicated in the key. The straight lines show the analytical results of Eq. (13) for comparison. The offset between the theoretical lines and the synthetic data is cosmetic (so the lines can be seen).
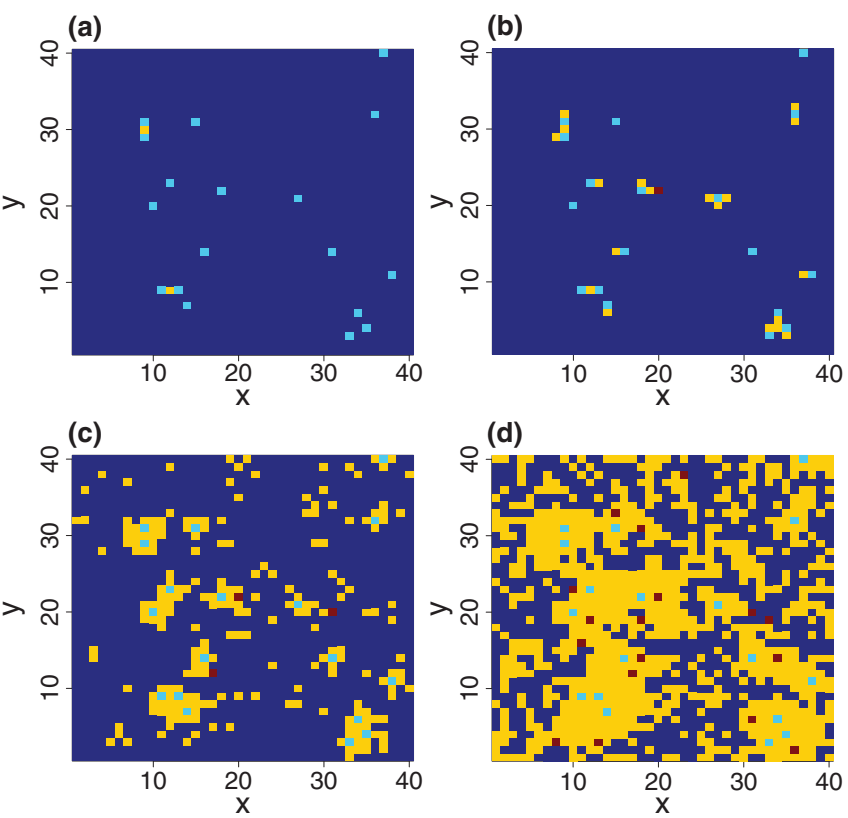

FIG. 4. (Color online) Subset of a FBM with $N=401 \times 401$, $W=0.1, a=1, \gamma=1$, and $\sigma=0.01$ after (a) 100, (b) 2000, (c) 20000 , and (d) 80000 broken fibers due to the aging process. Dark blue fields mark intact fibers, light blue fields mark fibers initially broken due to the applied stress, yellow fields mark fibers which broke due to the aging process (silent events), and red fields mark fibers which broke due to the local stress redistribution.

\section{CREEP FAILURE IN LLS FBM}

When the load is equally shared by all surviving fibers, the failure process does not have any dependence on the underlying lattice topology or on the spatial arrangement of broken fibers. However, when the load sharing is localized, spatial correlations emerge in the system which give rise to a more complex breaking process. Under a low external load $\sigma \ll \sigma_{c}$, a small amount of fibers initially break which are randomly scattered over the square lattice. Each fiber next to any broken fiber is carrying an additional stress, which enhances the aging process and thus leads to an earlier failure of this fiber. This is illustrated in Fig. 4, where fibers next to two broken neighbors break first [Fig. 4(a)], then the fibers with one broken neighbor break [Fig. 4(b)], and finally free fibers with no broken neighbors follow [Fig. 4(c)]. Only near failure time is the amount of AE increasing and broken clusters start to coalesce [Fig. 4(d)]. At sufficiently low loads, the fraction of initially broken fibers is so small that the fibers having at least one broken neighbor are distant from each other. Consequently, their failure process is completely uncorrelated. The onset time $t_{1, n}$ of such scenarios with $n=1$ or 2 initially broken neighbors of the considered fibers is controlled by the minimum value of the aging thresholds,

$$
t_{1, n}=\frac{1-W}{a\left(S_{n} \sigma\right)^{\gamma}} .
$$

Here, $S_{n}$ denotes the relative fraction of the local stress at relevant fibers with $n$ initially broken neighbors, which is $S_{2}=$ $6 / 4$ for scenario 1 (two initially broken neighbors) and $S_{1}=$ $5 / 4$ for scenario 2 (one initially broken neighbor). Due to the 
stress concentration, the aging-induced breaking sets on earlier in LLS than in ELS.

The interevent times for this case can be obtained as

$$
\tau_{i, n} \approx \frac{2 W}{R_{n} N} \frac{1}{a\left(S_{n} \sigma\right)^{\gamma}}=\text { const },
$$

with $R_{n}$ being the fraction of relevant fibers in the lattice with $n$ initially broken neighbored fibers in comparison to the total number of fibers $N$. It has the values $R_{2} \approx 6 \sigma^{2}$ for scenario 1 and $R_{1}=4 \sigma$ for scenario 2. Here, $\tau_{i, n}$ is constant, since the breaking of a fiber does not affect those fibers which are not adjacent to the broken one. As a consequence, the breaking time of the $i$ th fiber $i_{n}$ within both scenarios is

$$
t_{i, n}=t_{1, n}+\left(i_{n}-1\right) \tau_{n},
$$

which is equivalent to

$$
i_{n}=1+\left(t-t_{1, n}\right) / \tau_{i, n},
$$

with $t=t_{i, n}$ the elapsed time. Thus, the total damage $D_{n}^{L}$ increases linearly in time within both scenarios,

$$
\begin{aligned}
D_{n}^{L} & =d_{n}+i_{n} \\
& =d_{n}+1-\frac{(1-W) R_{n} N}{2 W}+t \frac{a\left(S_{n} \sigma\right)^{\gamma} R_{n} N}{2 W},
\end{aligned}
$$

where $d_{n}$ is the damage already present in the system at the beginning of the scenario [40]. The linear behavior of the damage at this stage is a consequence of the flat (constant) distribution of thresholds and the independence of the single breaking events. The damage rate follows as

$$
n^{L}(t)=\frac{d D_{n}^{L}}{d t}=\frac{a\left(\sigma S_{n}\right)^{\gamma} R_{n} N}{2 W},
$$

which is also constant.

Figure 5 presents the numerical results for the average damage $\langle D\rangle$ due to the aging process (dashed blue curves) and due to local stress redistribution (dotted red curve) as a function of time $t$. LLS simulations of a FBM were carried out with $N=401 \times 401$ fibers at two different load values $\sigma=0.002$ and $\sigma=0.01$. The amount of disorder $W$ and the parameters of the aging law were set to $W=0.1, a=1$, varying $\gamma$ between 1 and 3 . The occurrence time of a certain amount of damage was averaged over 1000 realizations of the system. The analytical result for the damage given by Eq. (18) has been fitted to the damage caused by aging with $d_{n}$ the only fit parameter (black full curve) for scenario 1 with two broken neighbors $(2 n)$ and scenario 2 with one broken neighbor (1n) [41]. Clearly, this analytical expression fits the real damage (dashed blue curve) very well.

In the final stage of the damage process, fibers again break without necessarily having any broken neighbors. In this scenario of free fibers, the onset time is defined in the same way as in the other scenarios,

$$
1-W=a \sigma^{\gamma} t_{1,0} .
$$

Comparing Eq. (20) to Eq. (4) in the low load limit, the onset time of free breaking fibers is the same for both LLS and ELS.

In the case of local load sharing, the stress is redistributed to the four nearest neighbors if one fiber breaks. These neighboring fibers already have internal damage (due to
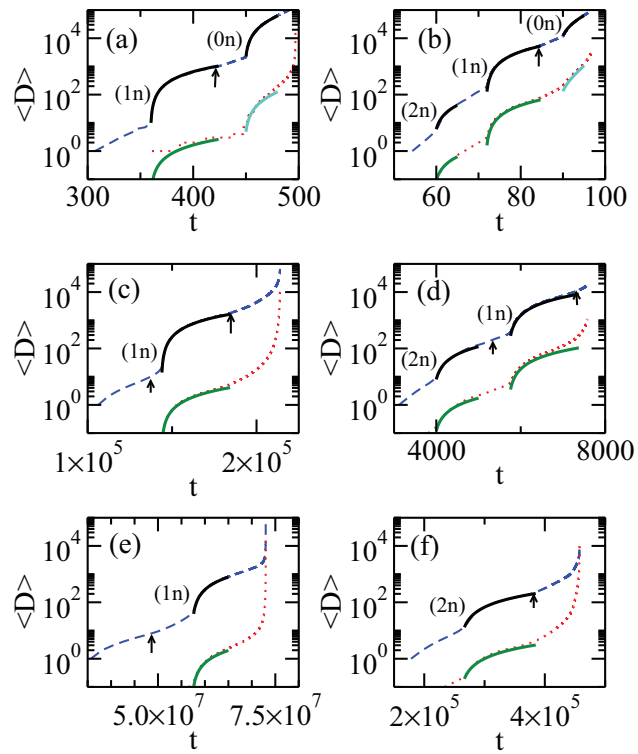

FIG. 5. (Color online) Average damage $\langle D\rangle$ due to the aging process (dashed blue curves) and due to local stress redistribution (dotted red curve) as a function of time $t$ for a FBM with $N=401 \times$ 401, $a=1, W=0.1$, and (a), (b) $\gamma=1$; (c), (d) $\gamma=2$; and (e), (f) $\gamma=3$, as well as $\sigma=0.002$ (left-hand side) and $\sigma=0.01$ (righthand side). The full black curves are fits according to Eq. (18) [for one (1n) or two (2n) broken neighbors] and Eq. (22) [for independent breaking ( $0 n)$ ] with fit parameter $d_{n}$ given by (a) $7(1 n), 435(0 n)$; (b) 6 (2n), $146(1 n), 2150(0 n)$; (c) 13 (1n); (d) 12 (2n), 279 (1n); (e) $38(1 n)$; and (f) $12(2 n)$. The green curves are the corresponding triggered damage according to Eqs. (28) and (29), and the turquoise curves are the triggered damage according to Eq. (30).

aging), $c=1-W+a \sigma^{\gamma}\left(t_{i, 0}-t_{1,0}\right) \approx 1-W+i_{0} \Delta c_{t h}$, for small $i_{0}$, where $i_{0}$ is the event number of the broken fiber within scenario $(0 n)$ and $\Delta c_{t h}$ is the distance of independent breaking thresholds, $\Delta c_{t h} \approx 2 W / N$. The question is now as follows: Does the local increase of the stress change the order of future events? The answer depends on the relative importance of stress enhancements controlled by $\gamma$ through the damage law given by Eq. (1) and of the amount of disorder represented by $W$. If $W$ is large and $\gamma$ is small, then the failure process is dominated by the disorder, i.e., the local stress increase enhances the breaking times of the neighbored fibers only slightly, but less than the time needed for overcoming $\Delta c_{t h}$, and hence the order is not changed. In this case, neglecting previously broken fibers, the next breaking fiber is carrying the stress $\sigma$ with probability $\left(N-5 i_{0}\right) /\left(N-i_{0}\right)$, and an enhanced stress $(5 / 4) \sigma$ with probability $4 i_{0} /\left(N-i_{0}\right)$. Thus, when averaging over different realizations of the system, the $i_{0}$ th breaking fiber is carrying the average stress,

$$
\sigma_{i, 0}=\sigma\left[\frac{N-5 i_{0}}{N-i_{0}}+\frac{4 i_{0}}{N-i_{0}} \frac{5}{4}\right]=\sigma \frac{N}{N-i_{0}} .
$$

The stress normalizes effectively, like in the ELS case [Eq. (6)], and as a consequence the damage evolves like in ELS. The interevent times are again given by Eq. (7), the breaking time of fiber $i$ is given by Eq. (8), and the time-dependent damage is given by Eq. (9) with $i=i_{0}$. For equal load sharing, the damage accumulation is accelerating right from 
the beginning of the creep process. However, when the load is locally redistributed, the acceleration phase, marked by the onset time of the breaking of free fibers, is preceded by a long stationary period. The stationary regime is characterized by a constant breaking rate, although it increases to a higher value when the system switches from scenario 1 to 2 .
In contrast to the ELS case, the LLS system already contains an initial number $d_{0}$ of failed fibers, which has to be added to the damage caused by the newly breaking fibers. Thus, the number of available fibers $N$ has to be replaced by $N-d_{0}$, and the stress $\sigma$ has to be replaced by the effective stress $\sigma N /\left(N-d_{0}\right)$, leading to

$$
D_{0}^{L}=d_{0}+i_{0} \approx N-\left(N-d_{0}\right)\left[1+\frac{(1-W)(\gamma+1)}{2 W}-t \frac{(\gamma+1) a \sigma^{\gamma}}{2 W\left(1-d_{0} / N\right)^{\gamma}}\right]^{\frac{1}{\gamma+1}},
$$

with $t=t_{i, 0}$ the elapsed time. The corresponding event rate is given by

$$
\begin{aligned}
n_{0}^{L}(t)=\frac{d D_{0}^{L}}{d t} & \approx \frac{\left(1-d_{0} / N\right)^{1-\gamma} N a \sigma^{\gamma}}{2 W}\left[1+\frac{(1-W)(\gamma+1)}{2 W}-t \frac{(\gamma+1) a \sigma^{\gamma}}{2 W\left(1-d_{0} / N\right)^{\gamma}}\right]^{-\frac{\gamma}{\gamma+1}} \\
& \approx \frac{\left(1-d_{0} / N\right)^{1-\gamma} N a \sigma^{\gamma}}{2 W}\left\{1-\left[\frac{t}{\left(1-d_{0} / N\right)^{\gamma}}-t_{1}\right] \frac{(\gamma+1) a \sigma^{\gamma}}{2 W}\right\}^{-\frac{\gamma}{\gamma+1}}
\end{aligned}
$$

which has a similar functional form to the ELS case, but with an enhanced time and, for $\gamma>1(\gamma<1)$, with an enhanced (decreased) prefactor. Thus when the stress is localized, the damage evolves faster than for equal load sharing in the parameter range $\gamma>1$.

If $W$ is small and $\gamma$ is large, the stress concentration around failed fibers has a dominating role in the rupture process. As a consequence, the order of breaking fibers changes, i.e., the affected fibers break earlier than before, while the unaffected ones still keep their original failure time. Since the number of affected fibers is increasing, the breaking process accelerates and the failure time is reduced in comparison to the case of large $W$ and small $\gamma$. This implies that the above expression (22) gives a lower bound for the effective time-dependent damage. In Figs. 5(a) and 5(b), the analytical result given by Eq. (22) has been fitted to the numerical values [black curves labeled with $(0 n)]$ and the corresponding values for $d_{0}$ are again given in the figure caption. Clearly, the analytical equation provides a very good description of the numerical results.

Figure 5 also shows that with increasing $\sigma$, the amount of triggered events (red dotted curve) is enhanced, and that with increasing $\gamma$, the time where the triggered events become relevant (in relation to the occurrence times for the different scenarios) is decreased. Thus, fewer of the scenarios discussed above would be observable from the remote measurements possible in a real experiment. Near failure time, the amount of triggered events is increased and the coalescence of existing damaged clusters becomes more and more relevant. Thus, close to failure, a higher amount of damage is expected than predicted by Eq. (22), as seen in Fig. 5.

A transient behavior can be observed between the different scenarios (Fig. 5), where the slope of the damage curve is steeper than the slope of the preceding scenario and lower than that of the following scenario. This may have different origins: (i) an averaging effect and/or (ii) the correlated damage growth which has been neglected in the derivations. The first effect occurs when, for different simulations, the same damage event may belong to different scenarios. Thus, this effect might be strong for early times where the overall damage is still small and thus fluctuations in the number of events within a scenario are large in comparison to the number itself. For example, there might exist some simulations in which the scenario of three initially broken neighbors occurs, whereas in other simulations, the largest number of initially broken neighbors is two. In this case, the first breaking fibers belong to different scenarios and thus their average occurrence time is something between the two scenarios. This effect should become less pronounced for later times, where the relative fluctuations of the event numbers within a scenario become smaller.

At these later times, the second mechanism dominates. If one fiber breaks, it redistributes its stress to the local neighborhood. These fibers then have an accelerated aging and will fail earlier than without the additional stress. Since this kind of failure is correlated to the breaking of the first fiber, we call this correlated damage. Thus, the transient behavior between scenario $2(1 n)$ and the scenario of free breaking fibers should be mainly the result of correlated failure triggered by the failure of events with initially one broken neighbor. Each event within scenario 2 redistributes the stress $(5 / 12) \sigma$ to its three remaining neighbors. These fibers will then carry the stress $(17 / 12) \sigma$, while before they only had the background stress $\sigma$. When the breaking time of the fiber out of scenario $2(1 n)$ is denoted by $t_{b}$, then the age of the three neighbored fibers is given by

$$
c=a \sigma^{\gamma} t_{b}+(17 / 12)^{\gamma} a \sigma^{\gamma}\left(t-t_{b}\right) \text {. }
$$

It follows for the correlated failure time $t_{\text {corr, } 1}$ of these fibers that

$$
t_{\mathrm{corr}, 1}=\frac{(12 / 17)^{\gamma}}{a \sigma^{\gamma}}\left\{c_{t h}+\left[(17 / 12)^{\gamma}-1\right] a \sigma^{\gamma} t_{b}\right\} .
$$

The failure times of these fibers depend not only on their local breaking thresholds $c_{t h}$, but also on the breaking time $t_{b}$ of the triggering fiber. The earliest breaking time of such a correlated event is thus given by $t_{b}=t_{1,1}=\frac{1-W}{(5 / 4)^{\gamma} a \sigma^{\gamma}}$ 


$$
\begin{aligned}
\text { and } c_{t h} & =1-W \\
t_{\min , 1} & =\frac{(12 / 17)^{\gamma}}{a \sigma^{\gamma}}\left\{1-W+\left[(17 / 12)^{\gamma}-1\right](4 / 5)^{\gamma}(1-W)\right\} \\
& =t_{1,0}\left[(12 / 17)^{\gamma}+(4 / 5)^{\gamma}-(12 / 17)^{\gamma}(4 / 5)^{\gamma}\right] .
\end{aligned}
$$

The value of the term in the square bracket in the last line is always smaller than 1 , which implies that there might always be a transient behavior between this and the next scenario. This onset time of correlated damage decreases with increasing $\gamma$ values. Consequently, the transient behavior (or deviations from linear damage growth for the uncorrelated breaking fibers) should occur earlier and hence be more pronounced for large $\gamma$ values. In Figs. 5(a)-5(d), these onset times are indicated by an arrow. They are in good agreement with the numerical results where the damage starts to increase nonlinearly. In Fig. 5(f), the crossover cannot be seen since the system failure occurs before the onset of scenario $2(1 n)$. In Fig. 5(e), scenario $2(1 n)$ is still observable, but also here global failure of the bundle occurs earlier than the transient time because of the increased amount of triggered events (red dotted curve).

The corresponding onset times of correlated damage growth for scenario $1(2 n)$ are given by

$$
t_{\min , 2}=t_{1,1}\left[(5 / 7)^{\gamma}+(5 / 6)^{\gamma}-(5 / 6)^{\gamma}(4 / 7)^{\gamma}\right] .
$$

The value of the term in the square bracket is not always smaller than 1 , e.g., its value is $15 / 14$ for $\gamma=1$. Thus, a transient behavior due to this correlated growth should be only visible for large $\gamma$ values. For small $\gamma$ values, the observed transient is more likely to be an averaging effect. The onset of this transient behavior is also indicated by arrows in Figs. 5(c)-5(f), where $\gamma$ is large enough for $t_{\min , 2}<t_{1,1}$. For $\gamma=3$ [Figs. 5(e) and $5(\mathrm{f})$ ], the numerical results are in good agreement with the analytical results. The averaging effect only plays a significant role where $\gamma=2$ in Figs. 5(c) and 5(d).

\section{TRIGGERED EVENTS IN LLS FBM}

Based on the above physical picture of the breaking process, we can also calculate analytically the amount of acoustic emissions. The calculations are restricted to the case where with each stress redistribution, only one fiber breaking is triggered at the same time step, i.e., we only consider avalanches of size 1 . This condition holds as an approximation in the low load limit for times far away from failure, where the overall damage in the system is still low, and thus the stress which gets redistributed is small.

If $n$ fibers increase their stress from $\sigma$ to $\sigma+\Delta \sigma$, then on average $n \Delta \sigma$ fibers will fail as triggered events since the breaking thresholds $\sigma_{t h}$ are uniformly distributed between 0 and 1. It follows for the different scenarios:

(i) If a fiber with two broken neighbors fails, then the two remaining neighbors increase their stress by the amount $\Delta \sigma=$ $(3 / 4) \sigma$. Thus, the amount of triggered damage is given by

$$
D_{t, 2}=2 D_{2}(3 / 4) \sigma=\frac{3 D_{2} \sigma}{2} .
$$

This analytical result is represented by the green curve in Figs. 5(b), 5(d), and 5(f), and it agrees very well with the numerical result (red dotted curve). (ii) If a fiber with one broken neighbor fails, then the three remaining neighbors will increase their stress by the amount $\Delta \sigma=(5 / 12) \sigma$. Thus, the amount of triggered damage is given by

$$
D_{t, 1}=3 D_{1}(5 / 12) \sigma=\frac{15 D_{1} \sigma}{12} .
$$

Again, this result is plotted as a green curve in Figs. 5(a)-5(e), where a good agreement can be observed with the numerical results.

(iii) If a free fiber fails, then the stress is normalized to $\sigma N /\left(N-i_{0}\right)$, yielding, for the $\left(N-i_{0}\right)$ remaining fibers, a stress increment of $\Delta \sigma=\sigma\left\{N /\left(N-i_{0}\right)-N /\left[N-\left(i_{0}-\right.\right.\right.$ $1)]\}=\sigma N /\left\{\left(N-i_{0}\right)\left[N-\left(i_{0}-1\right)\right]\right\}$. Thus, the amount of triggered damage is given by

$$
D_{t, 0}=\frac{D_{0} \sigma N}{N-\left(D_{0}-1\right)},
$$

under the condition that each triggered failure consists of only one fiber. This result is presented by turquoise curves in Figs. 5(a) and 5(b). Here again the numerical and the analytical results are in good agreement.

The strong increase of triggered events near the ultimate failure time which deviates from the above equations is mainly because here the triggered breaking of a fiber again triggers further breaking of neighbored fibers, and so on. As a consequence, large dynamic avalanches of arbitrary size are generated in this regime.

\section{DISCUSSION}

We analyzed the time evolution of damage in a fiber bundle model of heterogeneous materials subject to a constant external load. Fibers of the model undergo an aging process, which captures environmentally induced degradation of material elements. In order to reveal the role of the inhomogeneous stress field in the aging process, we considered the limiting cases of equal and localized sharing. Our study is restricted to low external loads where acoustic damage does not have a relevant contribution to the overall damage.

We found that the onset of aging-induced fiber failure occurs earlier for LLS than for ELS due to the stress concentration on intact fibers neighboring the failed ones. For equal load sharing, the cumulative damage has been calculated analytically as a function of time by mean field theory. These calculations revealed that due to the long range interaction, the system accelerates right from the beginning such that the event rate has a time-to-failure power-law behavior. The exponent proved to be dependent upon the parameters of the aging law.

When the load sharing is localized, the inhomogeneous stress field around broken fibers gives rise to a more complex time evolution: The breaking process remains stationary for a long duration; however, it is composed of segments of constant breaking rate which increase as spatial regions of the system with different stress concentration dominate the failure process. On the square lattice, the first fibers to break are those between two initially broken neighbors, and they break at a constant rate. In the second stage, fibers having one initially broken neighbor break, again with an on-average constant rate, but higher than before. In the last stage of the 
breaking process, acceleration sets on towards global failure when free fibers with no broken neighbors start to break with a rate which increases with time. As a very interesting outcome, we showed analytically that if the rupture process is dominated by the structural heterogeneity of the material, then the final acceleration to global failure is characterized by the same time-to-failure power law of the event rate as in the mean field limit of the system. Our discrete approach enabled us to derive the time evolution of small-sized triggered breaking events as well. The analytical results have been verified by computer simulations both for equal and localized load sharing.

Earth materials are typically subject to loads which are significantly smaller than their short-term fracture strength. Our study revealed that under such conditions, the time evolution of the system is mainly determined by the slowly advancing aging process, which also dominates the overall damage.
Since aging-induced silent damage cannot be monitored by standard nondestructive techniques such as acoustic emission, model calculations like ours are indispensable for reliability assessments.

\section{ACKNOWLEDGMENTS}

This work was supported by the European Commissions by the Complexity-NET pilot project LOCAT. D.Zs. and F.K. were supported by the Projects No. TAMOP4.2.2.A-11/1/KONV-2012-0036, No. TAMOP-4.2.2/B-10/12010-0024, No. TAMOP4.2.4.A/2-11-1-2012-0001, and No. ERANET-HU-09-1-2011-0002. The projects are implemented through the New Hungary Development Plan, co-financed by the European Union, European Social Fund, and European Regional Development Fund. F.K. acknowledges the support of OTKA K84157.
[1] M. Alava, P. K. Nukala, and S. Zapperi, Adv. Phys. 55, 349 (2006).

[2] B. K. Chakrabarti and L. G. Benguigui, Statistical Physics of Fracture and Breakdown in Disordered Systems (Oxford University Press, New York, 1997).

[3] D. Lockner, Int. J. Rock Mech. Min. Sci. Geomech. Abstr. 30, 883 (1993).

[4] H. Nechad, A. Helmstetter, R. ElGuerjouma, and D. Sornette, Phys. Rev. Lett. 94, 045501 (2005).

[5] S. Pradhan, A. Hansen, and B. K. Chakrabarti, Rev. Mod. Phys. 82, 499 (2010).

[6] F. Kun, F. Raischel, R. C. Hidalgo, and H. J. Herrmann, in Modeling Critical and Catastrophic Phenomena in Geoscience: A Statistical Physics Approach, edited by P. Bhattacharyya and B. K. Chakrabarti, Lecture Notes in Physics (Springer-Verlag, Berlin, Heidelberg, New York, 2006), pp. 57-92.

[7] D.-P. Hao, G. Tang, Z.-P. Xun, H. Xia, and K. Han, J. Stat. Mech. (2012) P10008.

[8] D. L. Turcotte, W. I. Newman, and R. Shcherbakov, Geophys. J. Int. 152, 718 (2003).

[9] I. Reiweger, J. Schweizer, J. Dual, and H. J. Herrmann, J. Glaciol. 55, 997 (2009).

[10] J. B. Rundle, D. L. Turcotte, R. Shcherbakov, W. Klein, and C. Sammis, Rev. Geophys. 41, 1019 (2003).

[11] S. Sadhukhan, T. Dutta, and S. Tarafdar, Physica A 390, 731 (2011).

[12] G. A. D’Addetta, F. Kun, and E. Ramm, Granular Matter 4, 77 (2002).

[13] H. A. Carmona, F. Kun, J. S. Andrade, and H. J. Herrmann, Phys. Rev. E 75, 046115 (2007).

[14] P. V. V. Nukala, S. Simunovic, and S. Zapperi, J. Stat. Mech: Theor. Exp. (2004) P08001.

[15] A. Petri, G. Paparo, A. Vespignani, A. Alippi, and M. Costantini, Phys. Rev. Lett. 73, 3423 (1994).

[16] C. Maes, A. VanMoffaert, H. Frederix, and H. Strauven, Phys. Rev. B 57, 4987 (1998).

[17] P. R. Sammonds, P. G. Meredith, and I. G. Main, Nature (London) 359, 228 (1992).

[18] S. Lennartz-Sassinek, F. Kun, M. Zaiser, I. Main, and Z. Danku, J. Phys. Conf. Ser. 410, 012064 (2013).
[19] F. Kun, M. H. Costa, R. N. C. Filho, J. S. A. Jr, J. B. Soares, and H. J. Herrmann, J. Stat. Mech. (2007) P02003.

[20] F. Kun, H. A. Carmona, J. S. Andrade, and H. J. Herrmann, Phys. Rev. Lett. 100, 094301 (2008).

[21] F. Kun, Z. Halász, J. S. Andrade, and H. J. Herrmann, J. Stat. Mech. (2009) P01021.

[22] Z. Halász, Z. Danku, and F. Kun, Phys. Rev. E 85, 016116 (2012).

[23] H. E. Daniels, Proc. R. Soc. London A 183, 405 (1945).

[24] B. D. Coleman, J. Appl. Phys. 27, 862 (1956).

[25] M. Kloster, A. Hansen, and P. C. Hemmer, Phys. Rev. E 56, 2615 (1997).

[26] B. Fyffe and M. Zaiser, Cold Regions Sci. Technol. 40, 229 (2004).

[27] M. Zaiser, P. Moretti, and A. K. a. E. C. Aifantis, J. Stat. Mech. (2009) P02047.

[28] R. C. Hidalgo, F. Kun, and H. J. Herrmann, Phys. Rev. E 65, 032502 (2002).

[29] W. I. Newman and S. L. Phoenix, Phys. Rev. E 63, 021507 (2001).

[30] A. Politi, S. Ciliberto, and R. Scorretti, Phys. Rev. E 66, 026107 (2002).

[31] N. Yoshioka, F. Kun, and N. Ito, Phys. Rev. Lett. 101, 145502 (2008).

[32] I. G. Main, Geophys. J. Int. 142, 151 (2000).

[33] M. Heap, P. Baud, P. Meredith, S. Vinciguerra, A. Bell, and I. Main, Earth Planet. Sci. Lett. 307, 71 (2011).

[34] In real experiments, not all dynamic events can be seen above background acoustic noise. So, in practice, a finite threshold of avalanche size above the elementary scale is needed.

[35] Y. Ogata, T. Utsu, and K. Katsura, Geophys. J. Int. 121, 233 (1995).

[36] A. Helmstetter, D. Sornette, and J. R. Grasso, J. Geophys. Res. 108, 2046 (2003).

[37] L. Faenza, S. Hainzl, and F. Scherbaum, Tectonophysics 470, 195 (2009).

[38] M. V. Rodkin, in Geophysical Monograph Series, edited by A. S. Sharma, A. Bunde, V. P. Dimri, and D. N. Baker (American Geophysical Union, Washington, DC, 2012), Vol. 196, pp. 27-39. 
[39] A. F. Bell, M. Naylor, M. J. Heap, and I. G. Main, Geophys. Res. Lett. 38, L15304 (2011).

[40] In [18], for $\gamma=1$, a different expression was derived for the damage in these early scenarios. There, the authors have mistakenly taken into account interactions between breaking fibers. But these scenarios are only dominant at early times far away from failure time, where the total damage is still small and even the (mistakenly) assumed interaction is weak. Thus, the expression in [18] can be approximated by a linear function in time, which is equivalent to Eq. (18) derived here.

[41] We do not give error bars for $d_{n}$ for the following reason. Although the dominating scenario in each individual simulation should usually be the same as for the averaged case, and with it the approximate event rate, this does not necessarily have to be the case for the initial preexisting damage for each scenario.
Even if we would have given a goodness of fit value for $d_{n}$ for the average case, this would not say anything about how good the same parameter would fit for the individual cases. The first two scenarios correspond to a linear damage increase. We have estimated the slope analytically and only the additive constant was fitted. That the chosen fits are acceptable can be seen in Fig. 5 for small damage values (small values on the $y$ axis). Here, due to the logarithmic plot, differences between the fitted and the numerical curve would be enhanced in comparison to large damage values where the relative difference might appear very small. Nevertheless, if it would be possible to measure these silent damage events in an experiment, one usually does not know the exact value of preexisting damage and one would measure the event rate. And, for the early scenarios 1 and 2, we have been able to determine this rate analytically without any fit parameter [Eq. (19) is independent from $d_{n}$ ]. 